\title{
Experimental Modelling of Czochralski Melt Flow with a Slow Crystal Dummy Rotation
}

\author{
V. Haslavsky, A.Yu. GelfGat* and E. Kit \\ School of Mechanical Engineering, Faculty of Engineering, Tel-Aviv University \\ Ramat Aviv, Tel-Aviv, 69978, Israel
}

\begin{abstract}
This study examines the effect of slow crystal dummy rotation on three-dimensional oscillatory instability and time-dependent supercritical flow states in a Czochralski melt flow experimental model. To enable further comparison with numerical modelling, the experiments are carried out using a $20 \mathrm{cSt}$ silicone oil as an experimental liquid and in a large diameter crucible, which allows one to work in a narrow temperature interval, so that temperature dependence of all the thermophysical properties of the experimental liquid can be neglected. The measurements confirm, partially qualitatively and partially quantitatively, earlier numerical predictions on destabilization ofthe Czochralski convective flow by a slow rotation. A simple power dependence of flow oscillations frequency on the Grashof and rotational Reynolds numbers that fits all the experimental runs was found.
\end{abstract}

DOI: $10.12693 /$ APhysPolA.124.193

PACS: 81.10.Aj, 47.55.P-, 44.27.+g

\section{Introduction}

This article presents an experimental study ofthe Czochralski type flows driven by buoyancy and thermocapillarity, and affected by a slow crystal rotation. These studies were started by Schwabe and his co-authors (see [1-3] and references therein), involving at later stages also our research group [4]. We continue to carry out experiments of this kind $[5,6]$, focusing, in particular, on producing of experimental data for validation of computational codes. Here we describe our first results extended to a larger experimental facility and more precise measurements. The experimental modelling of oscillatory instability onset in the Czochralski melt flow model, and the frequency characteristics of supercritical oscillatory flow regimes are presented.

We focus mainly on large Prandtl number melts, $\operatorname{Pr}>1$, where instabilities appear as spoke patterns, cold plumes, and oscillating jets [1-9]. These instabilities are well studied and are qualitatively described as results of the Rayleigh-Bénard mechanism that can be localized in different flow areas and, therefore, results in different patterns (see, e.g., $[6-9]$ and references therein). In this study we use viscous $20 \mathrm{cSt}$ silicone oil as a working liquid, and perform experiments in a relatively large experimental crucible, which allows us to work within a relatively narrow temperature gap of $5^{\circ} \mathrm{C}$, so that thermophysical properties of the fluid can be taken constant for the computational modeling purposes. As a result, we are able to confirm experimentally the numerically predicted effect of destabilization of the Czochralski convective flow by a slow rotation $[9,10]$, and to obtain certain quantitative agreement in comparison of experimentally

\footnotetext{
${ }^{*}$ corresponding author; e-mail: gelfgat@tau.ac.il
}

measured and numerically predicted frequencies of the flow oscillations.

The results presented below show rather novel and unexpected tendencies that, to the best of our knowledge, were never reported before, however yet to be verified against independent measurements and computations. First, we observe that at low rotation rates the instability sets in at approximately constant value of the Richardson number, which is the Grashof number divided by the squared Reynolds number. This implies that instability sets in when ratio of buoyancy to centrifugal forcing exceeds a certain value. Another novel observation is the power dependence of main oscillation frequency on the Grashof and Reynolds number that finalizes in a simple equation, which fits observations of all the experimental runs. It is stated again that the results are preliminary and will be subject to a thorough verification in our future studies.

\section{Experimental setup}

The experimental setup is sketched in Fig. 1 and is the same as described in [5], where the reader is addressed for details. Here we only mention that to mimic the Czochralski melt flow we study motion of experimental liquid in a transparent glass crucible whose sidewall and bottom are kept isothermal. The temperature difference is created by a cold and isothermal crystal dummy rotated by a separate DC motor. Thus, as in the Czochralski crystal growth system, the flow is driven by buoyancy, rotation and thermocapillarity. The crucible radius in our experiment is rather large, $45 \mathrm{~mm}$, which allows us to work at relatively low temperature differences. Thus, in the experiments described below the temperature difference did not exceed $5{ }^{\circ} \mathrm{C}$. Owing to such a small difference we were able to neglect dependence of the oil thermophysical properties on the temperature when performed computational studies for our setup. 


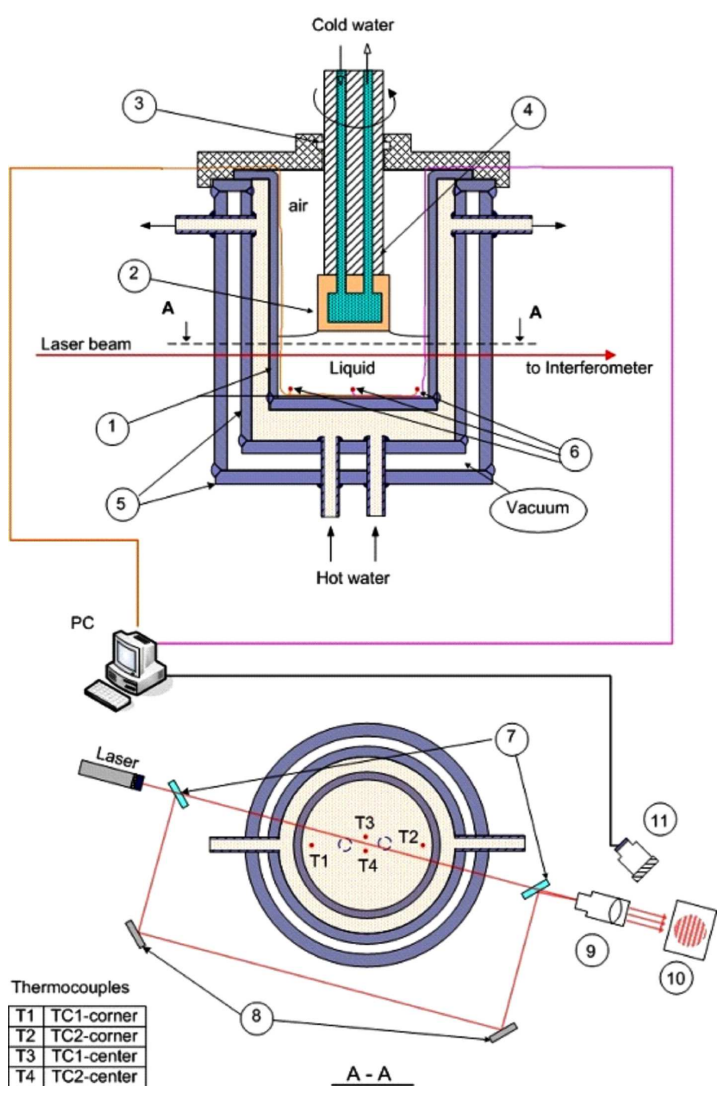

Fig. 1. Sketch of the experimental setup: (1) liquid container, (2) copper crystal dummy, (3) sealing ring, (4) pulling rod, (5) double-glass envelope, (6) thermocouples, (7) beam splitters, (8) mirrors, (9) beam expander, (10) screen, (11) camera.

TABLE

Thermophysical properties of $20 \mathrm{cSt}$ silicone oil.

\begin{tabular}{l|c}
\hline \hline \multicolumn{1}{c|}{ Property } & Value \\
\hline density $\left[\mathrm{kg} / \mathrm{m}^{3}\right]$ & 955 \\
kinematic viscosity $\left[\mathrm{m}^{2} / \mathrm{s}\right]$ & $0.2 \times 10^{-4}$ \\
heat diffusivity $\left[\mathrm{m}^{2} / \mathrm{s}\right]$ & $0.97 \times 10^{-7}$ \\
thermal expansion coefficient $\left[1 /{ }^{\circ} \mathrm{C}\right]$ & $1.07 \times 10^{-3}$ \\
surface tension coefficient $\left[\mathrm{kg} /\left(\mathrm{s}^{2}{ }^{\circ} \mathrm{C}\right)\right]$ & $-2.7 \times 10^{-5}$
\end{tabular}

Temperature fluctuations are measured simultaneously by very fine $0.1 \mathrm{~mm}$ diameter, T-type thermocouple wires, and by a Mach-Zehnder interferometer. All thermocouple signals and interferometer fringes are transmitted to PC for post-processing. The $20 \mathrm{cSt}$ silicon oil was used as experimental liquid. Properties of the silicon oil were taken from [11] and are listed in Table. The governing parameters are derived as $\operatorname{Pr}=206$, $\mathrm{Gr}=2391 \Delta T, \mathrm{Re}=101 \omega[\mathrm{rad} / \mathrm{s}], \mathrm{Ma}=658 \Delta T$, where $\Delta T$ is the temperature difference between the crucible wall and the crystal dummy, and $\omega$ is the angular velocity of the crystal dummy rotation. Since results here are reported for the single experimental liquid, the ratio $\mathrm{Ma} / \mathrm{Gr}=0.275$ is always constant, so that below we report mainly Grashof number values and dependences.

The series of experiments have been conducted for different crystal dummy rotation velocities varying between 0 and $5 \mathrm{rpm}$, with gradually changing temperature differences between 0 and $5.0^{\circ} \mathrm{C}$. The temperature fluctuations were measured at four different locations, so that a pair of thermocouples was installed near the axis and the crucible bottom and another pair in the crucible bottom-wall corner (Fig. 1).

Examples of temperature fluctuations measured by the thermocouples and the interferometer, as well as corresponding cross-verification of the results can be found in [5]. The temperature oscillations appear with a certain frequency and its harmonics, whose values are obtained via the Fourier transform of the temperature histories measured by thermocouples, or by oscillations of the amount of black pixels in a control area of an interferometry fringe. As shown in [5], both measurements yield identical frequency values. For the non-rotating dummy the strongest oscillations are observed in lower part of the crucible close to the axis. When crystal rotates we observe strong temperature oscillations also in the crucible lower corner. We are interested to measure a critical temperature difference at which oscillations appear, as well as dependence of the oscillation frequency on the governing parameters.

\section{Results}

Results for a slow dummy rotation on the instability onset are shown in Fig. 2. Measurements were carried out for dummy rotations with $\omega=1,2,3,4$, and $5 \mathrm{rpm}$ that corresponds to the Reynolds number varying between 10 and 53 . The temperature oscillations frequency is scaled by $\nu / R^{2}$, where $R$ is the crucible radius and $\nu$ is the oil kinematic viscosity. The temperature difference was varied between 0 and $5^{\circ} \mathrm{C}$ by the increment of $0.2^{\circ} \mathrm{C}$. This corresponds to variation of the Grashof numbers between 0 and 12000, and the Marangoni number between 0 and 3300 .

As follows from Fig. 2, a slow dummy rotation exhibits a destabilization effect, i.e. stability threshold shifts towards smaller temperature differences compared to the stationary dummy case. The first conclusion from Fig. 2 is the striking decrease of the critical Grashof number at relatively low rotation rates. At zero Reynolds number, i.e., when the crystal dummy is stationary, the critical Grashof numbers and oscillation frequencies are about an order of magnitude larger than those observed for the slow rotation $(20<\operatorname{Re}<50)$. This agrees with our earlier numerical predictions $[5,10]$. In agreement with these computations, a slow rotation destabilizes the convective flow starting from a certain small value of the Reynolds number. Thus, for $\omega=1 \mathrm{rpm}(\operatorname{Re}=10.5)$ the oscillation appear for $\mathrm{Gr}>7650$, while at $\omega=2 \mathrm{rpm}(\mathrm{Re}=21)$ the critical Grashof number is 480. The dashed line in Fig. 2 connects the points at which temperature oscillations appear, so that the flow is unstable below the curve 1 and 


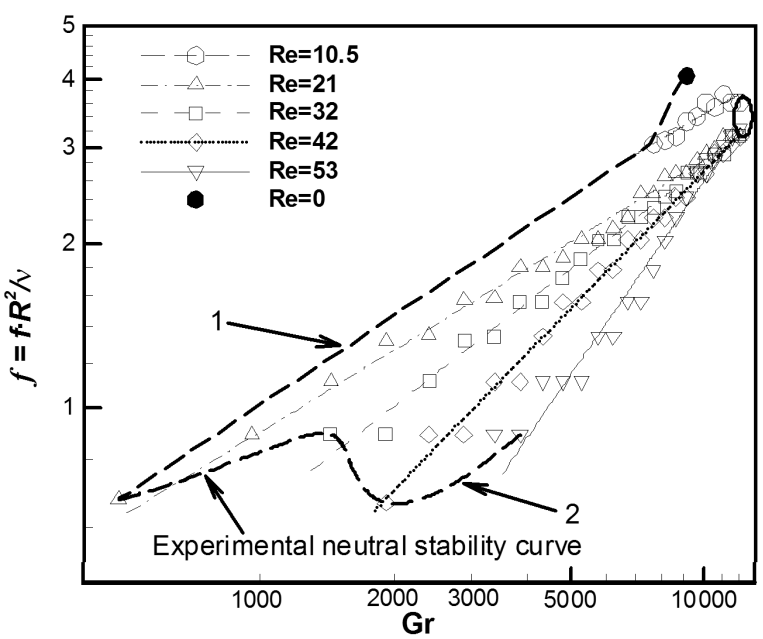

Fig. 2. Experimentally measured temperature oscillation frequencies as functions of the Grashof number at different dummy rotation rates. The straight lines represent the least square power fit of the points obtained at the same rotation rate.

above the curve 2 . The curve 1 corresponds to the mentioned above destabilization of convective flow by a slow rotation, while the curve 2 shows that at stronger rotation the critical Grashof number (or critical temperature difference) start to grow. The latter also qualitatively agrees with the numerical predictions. Further comparisons with the numerical results are given below.

Another interesting conclusion that follows from Fig. 2 is power dependences of the oscillations frequency on the Grashof number (temperature difference) observed at all five rotation rates with the gradually increased $\Delta T$. All the dependences were obtained as a least squares power fit assuming that the observed weak data scattering results from the experimental uncertainties. To the best of our knowledge, such dependences were never reported before. Furthermore, with the increase of the Grashof number all the power dependences converge to almost the same frequency value. The corresponding experimental points are enclosed into a small ellipse shown in Fig. 2. This possibly can be explained by a strong buoyancy effect that at large Grashof numbers makes the effect of weak rotation almost negligible.

The power dependence of the oscillation frequency on the Grashof number raises the question about existence of a similar dependence on the Reynolds number. This is really the case, as is illustrated in Fig. 3. As above, we observe that within relatively slow rotation rates considered here, power of the Reynolds number tends to zero, indicating that the flow is governed mainly by the buoyancy and, possibly, thermocapillary convection. By extrapolating the trends in Fig. 3 we observed that all the lines meet at almost same frequency value indicated by a small ellipse. We assume that all the power dependences observed belong to the same physical instability mechanism that starts at $\operatorname{Re} \approx 10$ and persists at small rotation

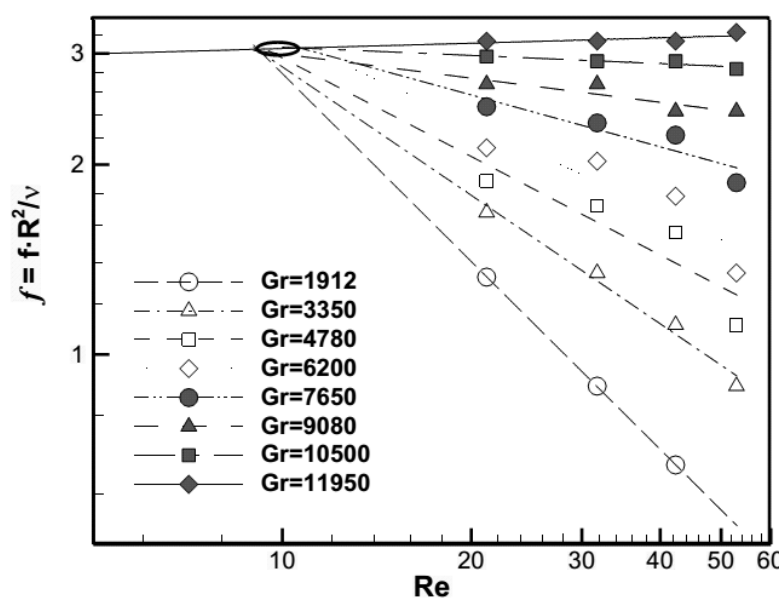

Fig. 3. Experimentally measured temperature oscillation frequencies as functions of the Reynolds number at different Grashof numbers (temperature differences). The straight lines represent the least square power fit of the points obtained at the same rotation rate.

rates at rather large interval of the Grashof number (temperature difference) values. At $\operatorname{Re}<10$ the instability is driven only by the thermal convection with a negligible effect of rotation.

Putting all the power dependences together we arrive to the following generalized formula:

$$
\begin{aligned}
& f(\operatorname{Re}, \mathrm{Gr})=\left(0.0032 \mathrm{Re}^{-3}-0.0024\right) \\
& \quad \times \mathrm{Gr}^{0.02 \operatorname{Re}+0.034} .
\end{aligned}
$$

This orderly correlation summarizes the power frequency dependences discussed above. Note that owing to the experimental range of low dummy rotations covered in this study, it describes only dummy rotation limited to $21<\operatorname{Re}<53$, and cannot be applied to the non-rotational case, while configurations with a faster rotation should be carefully tested prior application.

Figure 4 shows a new and striking observation that was never reported not only for the Czochralski model, but also for other flows driven by natural convection and rotation. Plotting all the measured frequencies against the Richardson number $\mathrm{Ri}=\mathrm{Gr} / \mathrm{Re}^{2}$, where zero frequency values correspond to the steady state flows, we observe that for all the rotation rates considered the oscillatory instability sets in at $\mathrm{Ri} \approx 1$. This constant value of the Richardson number implies an important conclusion: the observed instability sets in at a constant ratio of the buoyancy and centrifugal forces. At the same time it raises questions for further experimental and numerical studies. The first question is how this value of $\mathrm{Ri}$ varies with the Prandtl and Marangoni numbers. The second question is generality, i.e., in which Czochralski or other configurations and at which conditions the instability onset is defined by the critical value of the Richardson number? Is there a baroclinic instability mechanism as observed in rotating convective layers? All these and may be other questions are beyond the scope of the present 


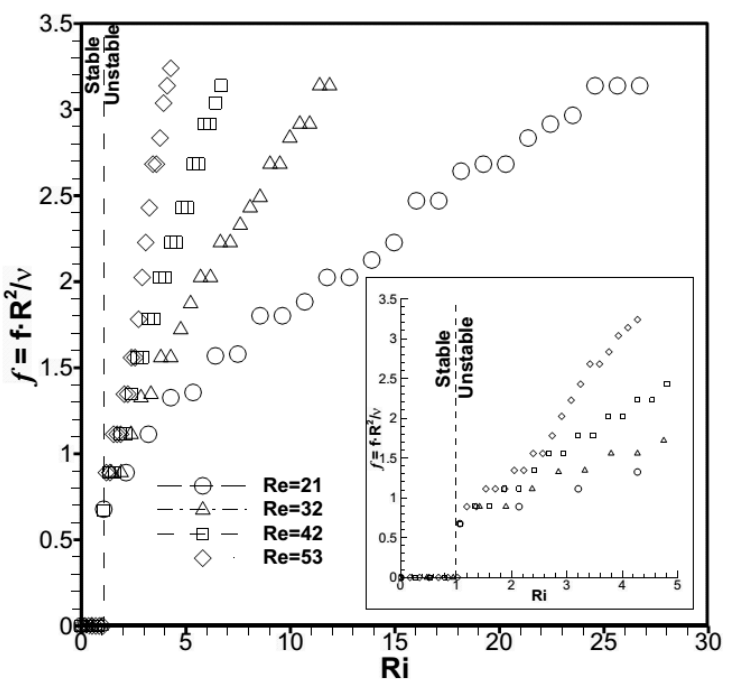

Fig. 4. Dependence of the measured oscillation frequency on the Richardson number at different Reynolds numbers. Black dashed line separates parameters corresponding to stable and unstable flows.

study and will be addressed in future.

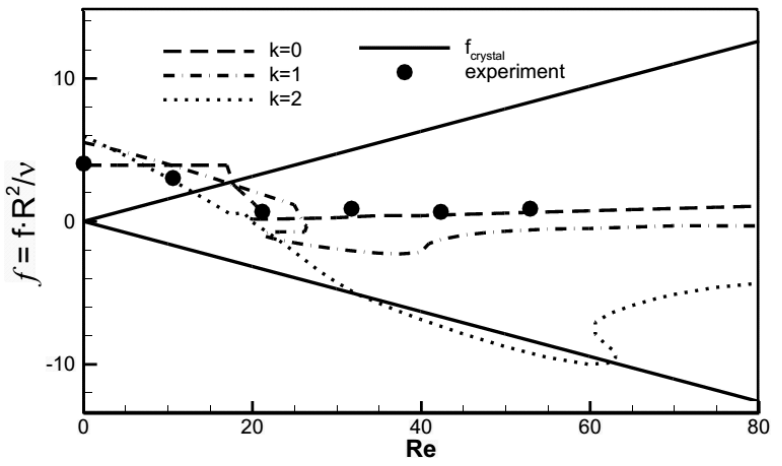

Fig. 5. Critical perturbation frequencies as functions of the Reynolds number. The curves show numerical results for different Fourier modes. The black circles correspond to the experimental results. The straight lines correspond to the crystal dummy rotation frequency.

In the following Figs. 5 and 6 we compare present experimental measurements with the numerical results on the oscillatory instability onset. Figure 5 shows the critical non-dimension perturbation frequency as a function of the Reynolds number. The curves represent results of numerical study for first three leading Fourier modes, $k=0,1$, and 2 , assuming that perturbations of a steady axisymmetric base flow are proportional to $\exp (\mathrm{i} k \theta)[10]$. The experimental measurements are shown by black circles. The solid straight lines correspond to frequency of crystal dummy rotation which increases linearly with the Reynolds number. Positive and negative frequency values correspond to the counter-clockwise or clockwise direction of crystal rotation, as well as show the direction

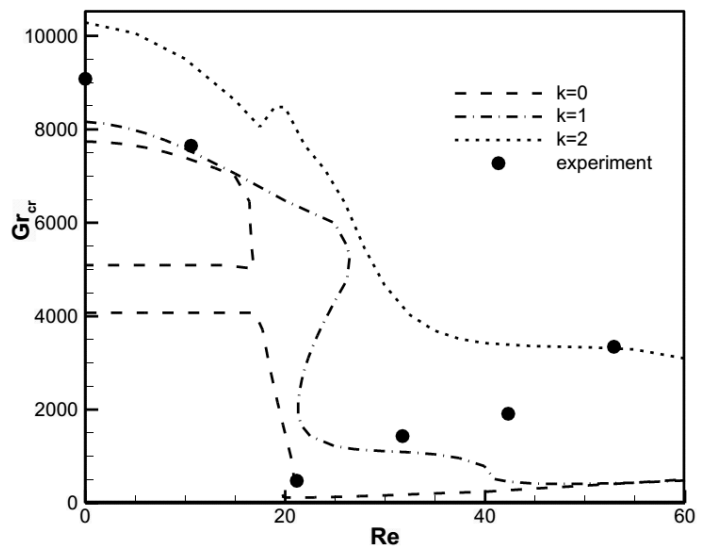

Fig. 6. Comparison of experimental and numerical results for the dependence of the critical Grashof number on the Reynolds number.

of propagation of three-dimensional azimuthal travelling waves. We observe a good agreement of experimentally measured frequencies with those predicted numerically for the axisymmetric $(k=0)$ Fourier mode. Two experimental points are close also to the frequencies of $k=2$ Fourier mode. However, since all the results are described by the same power dependence, we do not think that a switch of the eigenmode takes place. It is seen also from the next Fig. 5 that critical Grashof numbers of $k=0$ mode are noticeably smaller than that of the $k=2$ mode.

Figure 6 compares experimentally measured critical Grashof number with the numerical results on the flow linear stability. It is seen that conversely to comparison of the critical frequencies (Fig. 5), the agreement in critical Grashof numbers (critical temperature differences) is mainly qualitative. Mainly, the critical Grashof number steeply decreases when the rotational Reynolds number is gradually increased up to $\operatorname{Re} \approx 20$, and slowly increases beyond this value. This is in full agreement with similar observations of $[5,10]$. The explanation for the destabilization by a slow rotation is given in a recent study [9]. Basing on the above frequency comparison one would expect experimental point to lay along the $k=0$ numerical curve, however only two points out of six are close to this curve. This disagreement can be caused by different reasons, starting, e.g., from numerical accuracy. The oscillations predicted numerically for $\operatorname{Re}>20$ have very low frequency about $10^{-2} \mathrm{~Hz}$ (see Fig. 5), whose experimental measurement is not always possible. Also, the thermophysical parameters of the silicone oil, especially the temperature dependence of the surface tension, may be not very precise. It was shown in [4], for example, that a slight variation of the surface tension temperature coefficient can double the critical temperature.

We also observed that frequency of crystal dummy rotation appears in the frequency spectra. Usually, e.g., in [1], appearance of this frequency is attributed to the experimental imperfections. For example, a slight misalignment of the crystal dummy can lead to oscillations of 
the three-dimensional temperature field when the dummy rotates. In the present experiment, however we observe that in the interval $30<\operatorname{Re}<60$ the frequency of the $k=2$ Fourier mode is very close to the crystal rotation frequency (Fig. 4). It is seen also that at smaller Reynolds numbers, $10<\operatorname{Re}<20$, the frequencies of other Fourier modes are also very close to the frequency of crystal rotation. One can argue that small imperfections that always exist in the experimental setup can trigger the modes having frequency close to the dummy rotation one, so that the frequency of resulting non-linear flow becomes locked at the dummy rotation frequency value.

\section{Concluding remarks}

We have conducted a series of experiments in a Czochralski flow model setup using viscous 20 cSt silicone oil as a working liquid. Our purpose was to measure the steady-oscillatory transition in the flow governed by buoyancy, thermocapillarity and rotation, and to study the dependence of oscillations frequency on the governing parameters in non-linear supercritical flow regimes.

The experimental results allowed us to obtain two branches of the neutral stability curve, qualitatively supporting the numerically predicted destabilization of the convective flow by weak rotation (the first branch) and a slow growth of the critical temperature difference with further increase of the rotation rate (the second branch). Noticeably, the second branch is characterized by almost constant oscillations frequency.

A novel and quite unexpected observation shows that at all rotation rates considered the flow oscillations started at the same value of the Richardson number, $\mathrm{Ri} \approx 1$, implying that oscillatory instability is controlled by a certain balance between buoyancy and forced convection due to the dummy rotation. It is yet to be studied whether this effect retains at other Prandtl and Marangoni numbers, and how the Richardson number depends on these parameters.

Comparing the experimental and numerical results we find that the frequency of oscillations of supercritical flows compares much better than critical values of the temperature difference or the Grashof number. We argue that this discrepancy can be a result of numerical inaccuracies or not precisely known thermophysical properties. It should be noticed that numerically found instability limits correspond to a zero oscillations amplitude, while any experiment is capable to measure oscillations only starting from a certain finite amplitude.
At gradually increased supercriticalities, increasing either the Reynolds or the Grashof number, we found that frequency of temperature oscillations varies as a power of Re or Gr. Putting all the dependences together we arrived to Eq. (1). Again, it is yet to be explored whether these dependences exist in other Czochralski configurations, and at other, especially smaller, Prandtl numbers, as well as at larger Marangoni numbers. To verify these power dependences numerically one would need to perform a series of highly accurate fully three-dimensional non-linear time-dependent computations, which is a challenging computational task.

A very interesting and perhaps important observation at larger supercriticalities, corresponding to strongly non-linear regimes, is that all the power dependences of the oscillations frequencies on the Grashof number (the temperature difference) collided together, so that the result became independent of the Reynolds number, indicating that the resulting regime is governed mainly by the thermal convection.

\section{References}

[1] P. Hintz, D. Schwabe, J. Cryst. Growth 222, 356 (2001).

[2] P. Hintz, D. Schwabe, H. Wilke, J. Cryst. Growth 222, 343 (2001).

[3] D. Schwabe, J. Cryst. Growth, 237-239, 1849 (2002).

[4] M. Teitel, D. Schwabe, A. Gelfgat, J. Cryst. Growth 310, 1343 (2008).

[5] V. Haslavsky, E. Miroshnichenko, E. Kit, A. Gelfgat, J. Cryst. Growth 318, 156 (2011).

[6] V. Haslavsky, E. Miroshnichenko, E. Kit, A. Gelfgat, Fluid Dyn. Mater. Proc., submitted for publication.

[7] A.D.W. Jones, J. Cryst. Growth 63, 70 (1983).

[8] J.S. Szmyd, M. Jasczur, H. Ozoe, N. Imaishi, in: Studies of Flow Instabilities in Bulk Crystal Growth, Ed. A. Gelfgat, Transworld Research Network, Kerala (India) 2007, p. 179.

[9] A. Gelfgat, J. Fluid Mech. 385, 377 (2011).

[10] A. Gelfgat, in: Studies of Flow Instabilities in Bulk Crystal Growth, Ed. A. Gelfgat, Transworld Research Network, Kerala (India) 2007, p. 57.

[11] S. Rahal, P. Cerisier, H. Azuma, Exp. Fluids 43, 547 (2007). 